\title{
Ist ein Ganzkörperscreening überflüssig?
}

\author{
Müssen Teilnehmer am Screening auf Hautkrebs von \\ Kopf bis Fuß untersucht werden? Belgische Dermatolo- \\ gen haben eine Alternative hierzu getestet.
}

Das deutsche Hautkrebsscreening ist seit einigen Monaten ins Gerede gekommen. Teuer und nutzlos sei die Maßnahme, berichtete das ARD-Magazin „Kontraste“ und bezog sich dabei auf ein Gutachten, das dem Gemeinsamen Bundesausschuss vorliegen soll. Zwar seien etwa $25 \%$ mehr Diagnosen gestellt worden, aber die Sterblichkeitsrate sei nicht gesunken. Der Berufsverband der Deutschen Dermatologen (BVDD) sieht das anders. Vorstandssprecher Ralph von Kiedrowski argumentierte mit der steigenden Erkrankungsrate. Eine gleichbleibende Sterblichkeit bei erhöhter Inzidenz sei kein negatives Signal, sagte der BVDD-Sprecher.

Ein belgisches Forscherteam um die Dermatologin Isabelle Hoorens von der Universität Gent hat nun geprüft, ob sich ein alternatives Verfahren als Screeningmethode eignet. Hierzu wurden gut 9.000 Erwachsene in Ostflandern zu einem läsionsorientierten Screening (LOS) eingeladen. Zur Untersuchung erscheinen sollten Personen, die ein verdächtiges Mal aufwiesen.

Laut Definition waren das Hautveränderungen, die gemäß ABCD-Regel als auffällig gelten, als „hässliches Entlein“ (suspekt im Vergleich zum vorherrschenden Typ von Malen) imponieren, neu aufgetreten sind und länger als 4 Wochen bestehen oder gerötet sind und nicht abheilen. Eine Kontrollgruppe von ebenso vielen Personen wurde zum klassischen Ganzkörperscreening (GKS) gebeten.

Die Teilnahmerate erreichte $17,9 \%$ in der GKS- und 3,3\% in der LOS-Gruppe. Die Hautkrebs-Detektionsraten lagen bei 2,3\% (GKS) bzw. 3,2\% (LOS). Meist handelte es sich um Basalzellkarzinome. Umgerechnet auf die Zahl der Eingeladenen wurden beim GKS-Screening mehr Hautmalignome entdeckt (0,4 vs. $0,1 \%$ unter LOS). Das LOS dauerte rund 41 Sekunden, für das GKS wurden 232 Sekunden benötigt.

Im Unterschied zum deutschen Screening, an dem auch entsprechend geschulte Hausärzte beteiligt sind, wurden die Untersuchungen in der belgischen Studie nur von Dermatologen

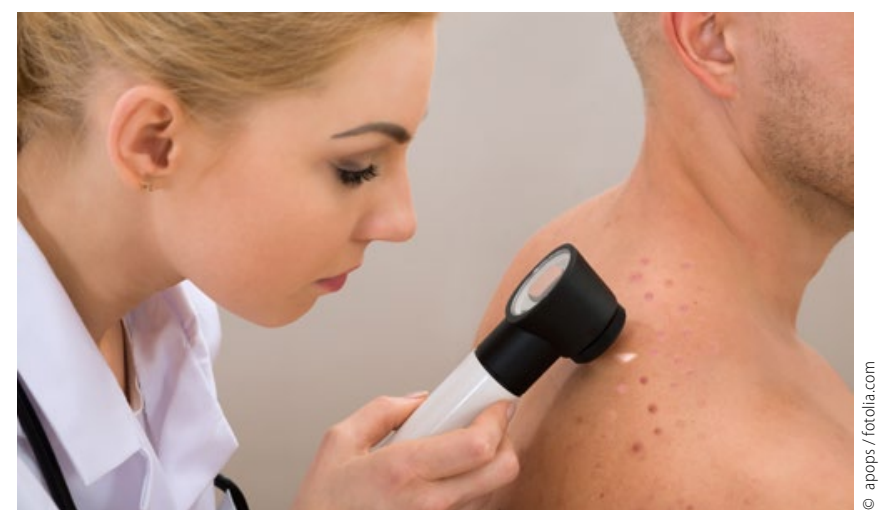

Läsionsorientiertes Screening oder Ganzkörperscreening?

vorgenommen. Damit erklärt sich für die Forscher jedenfalls zum Teil, weshalb ihre Detektionsraten (2,3 bzw. 3,2 \%) deutlich höher lagen als die in deutschen Studien errechneten $(0,8 \%)$.

\section{Alternative zum Ganzkörperscreening}

Grundsätzlich halten die Belgier das LOS bei langen Wartelisten und schmalen Budgets für eine akzeptable Alternative zum GKS, sofern es in der Obhut von Dermatologen liegt. Sie verweisen darauf, dass ein an das LOS anschließendes GKS bei unauffälliger Indexläsion nur selten $(0,4 \%$ in der Studie), bei suspektem Mal aber häufig (30\%) zu weiteren Befunden führt. Daher sei ein GKS vermutlich vor allem in solchen Fällen indiziert.

Allerdings sei es wichtig, die Teilnahmerate am LOS und damit die Gesamtzahl der entdeckten Hautmalignome zu erhöhen. Dabei müssten Unterschiede im Bildungsgrad und im Bewusstsein für das Problem Hautkrebs bei den Eingeladenen berücksichtigt werden, um eine Benachteiligung sozioökonomisch schlechter gestellter Gruppen, wie sie gegenwärtig auftrete, zu vermeiden.

Dr. Robert Bublak

Hoorens I et al. JAMA Dermatol 2015, Oct 14; doi:10.1001/jamadermatol.2015.2680

\section{Hautkrebsscreening}

\section{Wann die Dermatoskopie keine IGeL ist}

Bei der Früherkennungsuntersuchung auf Hautkrebs kann die Auflichtmikroskopie zum Einsatz kommen.

Wünscht der Kassenpatient die Dermatoskopie, muss er sie als IGeL bezahlen. Doch es gibt eine Ausnahme.

Seit 1. Juli 2008 haben gesetzlich Versicherte ab 35 Jahren alle zwei Jahre Anspruch auf eine Früherkennungsuntersuchung auf Hautkrebs, das sogenannte Hautkrebsscreening. Im Zusam- menhang mit dieser Leistung tritt immer wieder die Frage auf: Gehört die Dermatoskopie (auch Auflichtmikroskopie genannt) zum Hautkrebsscreening? 